\title{
Keeping large animals contained
}

\author{
Caring for lab animals is always a priority. But some, depending on the research they are being used for, can require \\ extra attention. As the animals in question get bigger, the task of keeping them in contained facilities can just get a \\ bit more challenging.
}

\section{Ellen P. Neff}

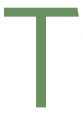
he discovery was a bit of serendipity, says Christopher Tuggle, a researcher at Iowa State University. Twenty years ago, a founder population of Yorkshire pigs, a breed widely raised in the United States for agriculture, was established at Iowa State for a feed efficiency project. Fast forward a few generations, and a litter was born that really packed on the pounds. Curious about what the animals were giving up to gain weight from fewer calories than other piglets, the research team at Iowa State trucked some animals down to collaborators at Kansas State for stress testing. A few didn't survive the journey, while a couple others quickly succumbed to a common porcine virus that's not usual lethal. Something was up with their immune system.

Had the animals died in Iowa, no one would have thought much of it-"a few piglets lost from a litter isn't necessarily something that we'd do research on," says Tuggle. But when the team in Kansas performed necropsies on the animals, they were indeed severely immunocompromised ${ }^{1}$. They had been initially protected by antibodies produced by their mother but could not mount immune responses on their own. Perhaps not a valuable feature in an agricultural animal, but Tuggle saw potential for a new biomedical model: a severe combined immunodeficient, or SCID, pig.

Such an animal might be used to study the deficiency itself, or as a recipient of human cells. A deficient immune system means the animals won't reject 'foreign' tissues as readily as one that has full defenses in place, but it also means they must be carefully protected from exposure to external pathogens that their handlers or environment might inadvertently expose them to.

Those who care for and work with laboratory animals, whether large or small, keep a close eye on the health of their charges. But some animals, such as the emerging SCID pig, gnotobiotic subjects, or those used in infectious disease research, can require higher levels of biocontainment. The larger the animal, the greater the challenge it can be to keep the outside out, or the inside in.

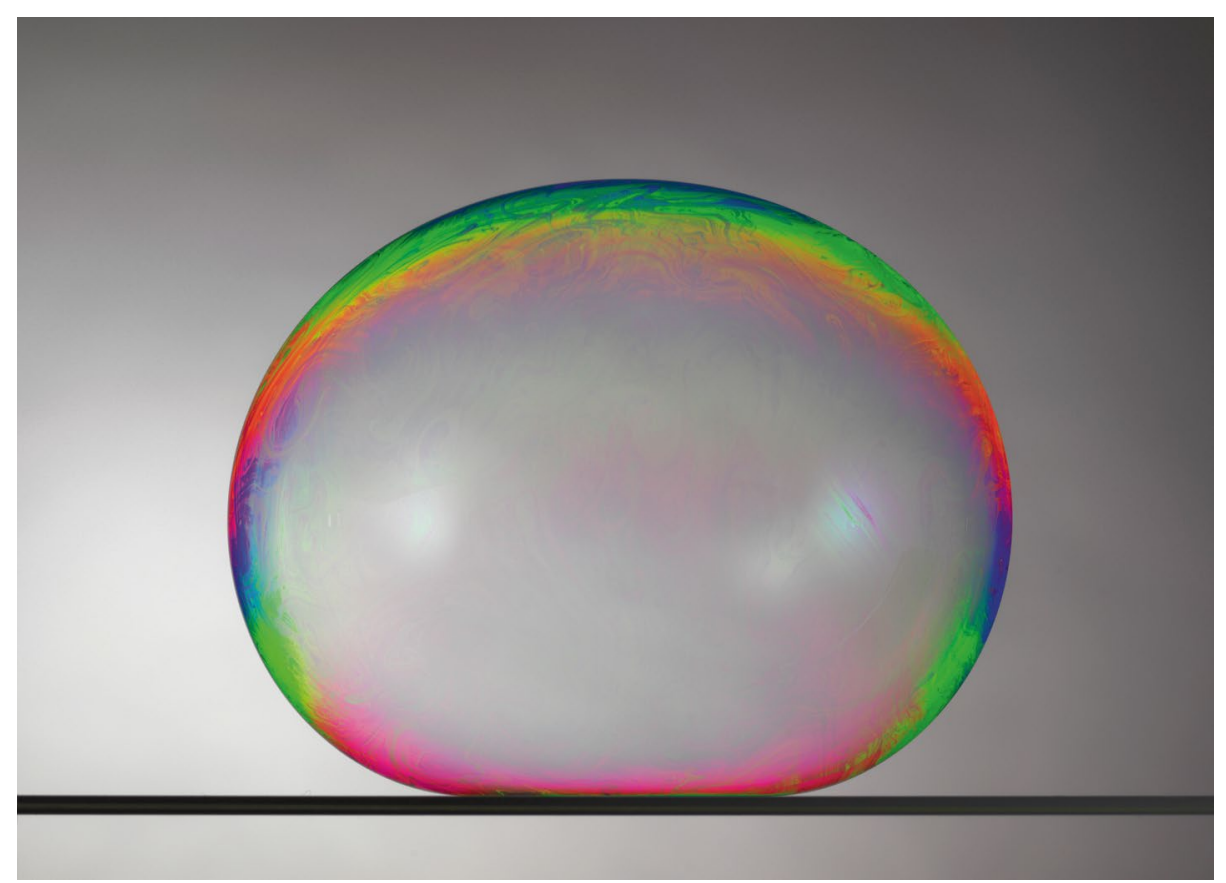

In the bubble: Specialized facilities and equipment are needed to keep large animals 'clean.' Credit: Bruno Gori / Cultura / Getty

\section{Keeping the outside out...}

The world is awash with microbes; some are benign, others potentially pathogenic. Their presence or absence can be an important consideration when working with animal models. Research animals can be raised and monitored such that they are specificpathogen free, but they still host various other microbes with less well-known identifies and potential impacts on a study. To know exactly what microbes are present, some researchers rely on gnotobiotic animals. These are animals with 'known' 'life' living on and within them. To keep them that way, they must be kept carefully isolated from the outside world and only exposed to the particular microbes the researchers want to explore.

A challenge, but not necessarily a new one: the gnotobiotic field emerged in the $1950 \mathrm{~s}^{2}$, when researchers first established germ-free derivation strategies and the sterile, filtered-air isolator units in which the animals will live. Over the years, there have been a number of gnotobiotic animals of various sizes: rodents, chickens, pigs, canines, and even calves - these are limited, however, to the first few weeks of life. Once a calf, a ruminant animal, develops the fourchambered stomach it needs to continue growing, maintaining germ-free status is no longer possible. Rodent facilities are becoming more common ${ }^{3}$, but those capable of maintaining the meticulous microbial status of larger animals remain fewer and farther in between.

It all has to start before the animals are even born. As a pregnant female nears term, she must undergo a hysterectomy; after which, neonates are carefully delivered into a sterile environment and transferred to their isolator unit. Such derivation can be relatively straightforward in mice-Michael Oglesbee, a researcher at Ohio State who 


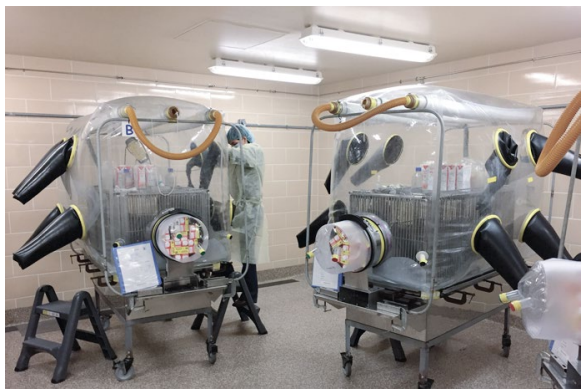

Gnotobiotic piglets are kept in large isolator units. Credit: M. Oglesbee

oversees the gnotobiotic pig facility at the University's Columbus campus, notes that principal investigators can often handle the necessary rodents steps themselves. Working with a pregnant sow to obtain gnotobiotic piglets, however, is a much bigger undertaking. A veterinary surgeon and anesthetist must be present for the procedure, along with trained staff that can deliver and transfer the piglets without contaminating them with any microbes. "You easily will have four technicians during the derivation," he says, "And then we go from derivation to maintenance."

Once comfortably in their isolators, the gnotobiotic piglets themselves aren't all that different from conventionally raised counterparts, says Oglesbee. Their space is restricted to the isolator though; their milk must be sterilized, and staff can only interact with them through gloves into the isolator units. Anything that must go into the unit, whether to care for the pigs or for research purposes, is sterilized in an antechamber before being transferred in to the isolator itself.

Most gnotobiotic piglets only spend a few weeks in an experiment and thus only grow to a few kilograms. But the equipment and staff needed to care for them is much more costly than for rodents. At Ohio State's gnotobiotic mouse facility, for example, the mice are kept in cages on an isolator rack, says Oglesbee. "The primary investment is the rack system," he says, along with a biosafety cabinet. For the pigs, multiple larger isolators might be needed if the program wants to work with multiple litters at time, as well as a large enough autoclave capable of cleaning those units after an experiment is over.

Immunodeficient animals must also be protected from the outside world. Without an intact immune system, they cannot mount immune defense against potential pathogens. All food and materials that come in contact with them must be carefully sterilized, and it's best they inhabit a sterile environment with filtered air too. But one advantage of an animal such as pig is its human-like size as it grows; an isolator unit would have to be very big indeed.

Tuggle and his colleagues received NIH funding to characterize the SCID pigthey published details about the genetic mutations the animals carry in $2015^{4}$-and to figure out how best to care for them. A conventional farm certainly wouldn't do, nor did the animals thrive in isolated animal rooms. Instead, they've turned to a customized biocontainment space, built by Fort Collins-based bioBUBBLe, Inc. Rather than constructing an entirely new building, the 'biobubble' can be installed in an existing space. Iowa State currently has three different bubbles for their pigs. The first, the 'entry bubble,' accordingly to Tuggle, is about 150 square feet and can house two litters of piglets while the team determines their health status. From there, a slightly roomier, 900 square foot bubble contains research pens as well four farrowing units that can be used to breed animals directly into the bubble itself via C-section from a thoroughly cleaned carrier sow. A third bubble now offers additional research pens for external scientists interested in working with the SCID animals but without their own bubble facilities. The bubbles receive HEPA-filtered air and are positively pressured, incoming water is irradiated and filtered, and all feed is gamma irradiated to destroy any incoming pathogens. Personnel must wear PPE.

With bubbles built, staff have been working on the best breeding and husbandry practices for the animals ${ }^{5}$. Space can be a little cramped says Tuggle, but, excluding the need to keep pathogens away, the pigs are just like any other pig. Staff interact with them twice a day, which comes with an abundance of marshmallows during clicker training sessions.

Currently, the animals can survive to about five or six months of age; by this point they will be about human-sized, but

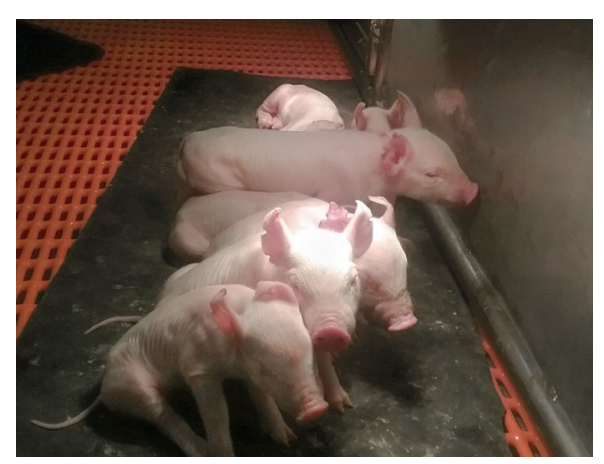

SCID piglets in lowa State's biobubble facility Credit: C. Tuggle
Tuggle and his colleagues are still looking to improve that. 'It's definitely early days in terms of really the best husbandry procedures for these very delicate animals," says Tuggle.

\section{... and keeping the inside in}

In the case of gnotobiotic and immuno deficient animals, the goal is to keep them from being exposed to unwanted microbes. But for other lines of research, it's important to keep what's in the facility from getting out. Studying infectious disease can require special containment measures, and large animals can be particularly valuable for virology and vaccine development ${ }^{6}$. But as animals get bigger, so do some of the daily challenges to caring for them.

The animals can be big indeed. To study a disease such as MERs, endemic in camels, you might need to bring in a camel. Livestock are studied as more translationally relevant large animal models for human research than smaller options such as rodents, but they are also used to understand and prevent agricultural diseases.

Joe Garthewaite's day at the Pirbright Institute in Surrey, England usually starts early. Garthwaite supervises the high containment, large animal isolation facility at the Institute, which is dedicated to infectious disease research, in particular of agricultural animals. The currently facility has existed for about 10 years and consists of a series of rooms connected by a 'clean' corridor; entry and exist is via airlocks. Engineering and biosafety teams keep a close eye on building systems such as the air handling system and temperature control, while animal technicians mind the animals inside.

Garthwaite's charges are all livestock, considerably larger than the average lab mouse: chickens, goats and sheep, pigs, and juvenile cattle that nonetheless can tip the scales at over 200 kilograms. The animals themselves come from commercial farms but are brought into the high containment facility to study various viral infections. The way they are cared for doesn't differ drastically from conventional settings-the animals are stilled checked on and fed twice a day-it just requires a bit more planning.

The day usually starts with discussions among the team of who needs to be in what room when. "You're in a building that has restrictions on what doors you can open," says Garthwaite, to keep infectious agents contained in designated rooms among designated animals. All food and equipment that might be needed must be accounted before going in, and anything coming out of the rooms must either thoroughly decontaminated or else incinerated without 


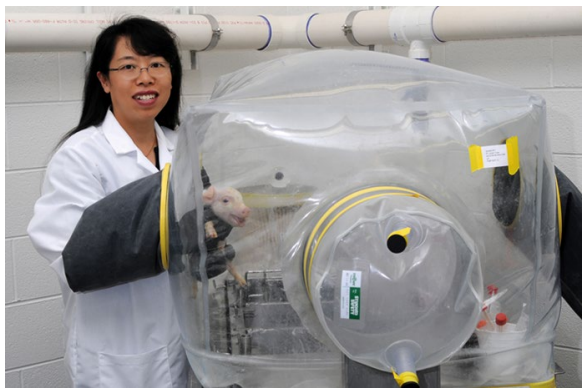

Lijuan Yuan holding a gnotobiotic piglet.

Credit: L. Yuan

leaving the facility-that includes all animal waste. Staff wear PPE, but all cleaning is done by hand, lest a power washer aerosolize viral particles. Training to work there can take upwards of six months.

Enriching the animals' Iives is a priority, as they otherwise inhabit a barren, poured concrete room during the course of the research in which they are being used. Pigs for example are provided with various toys as well as boxes to encourage natural rooting behavior; goats, which appreciate the opportunity to look around above their fellows, are given steps to stand on. CCTV cameras monitor the animals 24/7, and staff have been reviewing that footage of animal behavior to identify ways they might refine animal care further. "It's trying to put all the pieces of the jigsaw together to get a better understanding of how what we're doing influences the animals," says Animal Services lead Tony Smith. Room sizes are fixed currently, but Smith says they are planning for larger rooms for the larger animals in a new facility the Institute is designing.

\section{Drawing on big blank slates}

Protective gear donned and extensive checklists checked, the staff that care for such animals in tightly contained facilities do so in support of research questions that could not be asked any other way. Lijuan Yuan, a professor at Virginia Tech, relies on gnotobiotic piglets to study rotavirus, a viral infection that remains a leading cause of severe childhood diarrhea around the world. Neonatal pigs can be infected with the same viral strain that affects children, but there's also a potentially confounding porcine rotavirus that is hard to keep from infecting the animals unless they are kept in extremely clean environments. "A gnotobiotic environment is the only way you can study [the human rotavirus in] the pig model," Yuan says.
The gnotobiotic facilities at Ohio State (one at the main campus in Columbus, and a second in Wooster) were originally intended to study microbial pathogenesis free from the confounding influence of other microbes, says Oglesbee. Although the animals have intact immune systems, they can also be more susceptible to pathogens when they are germ-free; whereas in a conventional population only a few animals out of many infected might develop symptoms of a disease, working with gnotobiotic animals increases the odds. "You can get more robust results with a defined number of animals, so you use less animals," he says.

The gnotobiotic field too is shifting as interest grows in the microbiome. "We very much appreciate the role that microbial communities play in educating the immune system," Oglesbee says. "The question is now, what components of the microbial communities in the gut, on the integument, in the respiratory tract are having these effects." The relationships that guide susceptibility or resilience to a disease are more complex than one bad bug alone. "This is where a germ-free animal is important because you can actually define the microbial community," he says. "You have to start with a blank slate."

"Gnotobiotic pig programs are relatively simple in concept, but require detailoriented staff to be maintained and to function properly," Yuan writes in a recent chapter ${ }^{7}$ describing her experience setting up such a facility at Virginia Tech. Though in North America there are only six such facilities, she says she expects to see more as demand for large animal options to study the effects of the microbiome increase. Oglesbee is waiting to see the shift to large animal microbiome models really start to take off, but he expects it any day. Besides the cost associated with a gnotobiotic pig facility, there are more limited reagents available to work with pigs, relative to mouse. But growing interest in the animals may encourage that development as well.

The SCID pigs are a similar blank slate, from an immune system perspective. "We're placing the pig between the human and the mouse," says Tuggle, "The pig could be a very useful model before you go into actual clinical trials." Iowa State is actually on SCID pig version 2 . The first had mutations in a DNA repair pathway required for the production of $\mathrm{B}$ and $\mathrm{T}$ cells, but the animals still produced Natural Killer cells that could potentially interfere with human cells. They've since created animals with a second mutation that rids the pigs of such lymphocytes entirely, with the hopes to 'humanize' the animals in the near future.

There's interest in the animals for a number of questions. Collaborators from the University of Alabama for example are interested in studying pigs with an immune system derived from humans with Sickle Cell disease; others, such as a team at the Mayo Clinic (who Tuggle has been advising in the eventual construction of their own biobubble) want to study cardiac regeneration. The pig is a human-sized model in which to test whether human stem cells can be used to repair damaged cardiac tissue. Recently, the SCID pigs were used to test ways to improve skin engraftments. They might also make an interesting addition to pathogenesis studies-a particular type of immune cell of interest might be selected returned to the pig, Tuggle suggests. In a recent flu study, the SCID pigs eventually died but with less lung damage than immune-competent animals. With no adaptive immune system to summon the cells that cause inflammation-and, if gone on too long, tissue damage-the SCID pigs were able to evade that phenotype.

The animals need not only be used for direct human benefit. Many of the livestock used at Pirbright are being studied to benefit agricultural animals. A cohort of pigs has been used to help identify new details about African Swine Fever ${ }^{8}$, a hemorrhagic porcine disease; an outbreak in China is threatening millions of pigs there ${ }^{9}$. Cattle are used to study food-and-mouth disease. The institute also works with highly contained mice, but livestock are the ultimate recipients of any line of research.

The larger the animal, the larger the undertaking for those tasked with its care. Extra containment requirements brings extra challenges, but the research payoff can potentially be a big one.

\section{Ellen P. Neff \\ Lab Animal. \\ e-mail:ellen.neff@us.nature.com}

Published online: 19 September 2019 https://doi.org/10.1038/s41684-019-0403-x

\footnotetext{
References

1. Ozuna, A. G. et al. Vet Pathol 50, 144-146 (2013).

2. Reyniers, J. A. Ann NY Acad Sci. 78, 3-16 (1959).

3. Mallapaty, S. Lab Anim (NY) 46, 373-377 (2017).

4. Waide, E. H. et al. J Immunol 195, 3171-3179 (2015)

5. Powell, E. J. et al. Lab Anim 52, 402-412 (2018).

6. Gerdts, V. et al. ILAR J 56, 53-62 (2015).

7. Yuan, L., Jobst, P.M. and Weiss, M. Chapter 5 in Gnotobiotics (Schoeb, T.C. and Eaton K.A., eds.)Academic Press, Elsevier, 2017 8. Netherton, C. L. et al. Front Immunol. https://doi.org/10.3389/ fimmu.2019.01318 (2019).

9. Mallapaty, S. Nature 569, 13-14 (2019).
} 